Euthanasia

\section{Should euthanasia be legal? An international survey of neonatal intensive care units staff}

\section{R W Cooke}

\section{A commentary on the paper by Cuttini et al}

T he members of the EURONIC group have investigated the attitudes and practice of nurses and doctors with regard to euthanasia in neonatal units throughout Europe. Not surprisingly, they have found a wide range of beliefs and actions concerning an act that remains illegal for the newborn in all the countries studied. The group begin helpfully by defining what they mean by euthanasia, as debate is often confused by different understandings of what it means. Definitions range from "ending life...as by lethal injection or the suspension of extraordinary treatment" to "act of killing painlessly (especially one suffering from an incurable illness)". In the United Kingdom especially, doctors often distinguish between "active" and "passive" euthanasia (the latter involving withdrawal of intensive care), despite ethicists denying a difference between these actions if the intention is to end life. Those supporting withdrawal of care in selected cases argue that their intention is to reduce suffering by terminating ineffective treatment. The near universality of deaths following these decisions on neonatal units suggests that this may not be entirely true.

This study refers to "active" euthanasia. A wide variation of beliefs was shown, with those least in favour of a change in the law regarding euthanasia for selected newborns being predominantly female, religious, and more experienced in neonatal care, and those supporting change more likely to be childless researchers who had already participated in neonatal euthanasia.

It was perhaps not surprising to see little support for change in conservative Roman Catholic countries such as Italy, and more enthusiasm in countries such as the Netherlands with a tradition of more open government and public involvement in decision making, and already provision for euthanasia for older people. The most remarkable findings concerned French neonatologists and nurses who appeared to both believe in and practice active euthanasia in the newborn more than in any other European country. Their opposition to a change in the law was based on a concept that such actions were subjected to professional codes and outside the law. Even the involvement of parents was seen as undesirable because they might act in their own interests rather than those of the infant. Introducing a law would make doctors less careful about end of life decisions. Such arrogance has been seen in recent UK decisions involving pathologists and paediatricians. Commendably, the French National Ethics Committee for Health and Life Sciences has made recommendations for greater involvement of parents and more open decision making.

The authors lament that the extent of variation in belief and practice will rule out the development of uniform legal procedures covering neonatal euthanasia throughout Europe. This applies to other areas of legislation where personal beliefs, formed by local ethical values, custom, and teaching are involved. It is appropriate that the law should reflect these, and variation should occur, until perhaps a time comes when Europe is a single country with a single belief system; not very likely or even desirable in the near future. However, with improved communications and media exposure, consensus at least at the level of principles for management of the end of life of selected newborns, along the lines of the document of the ethics committee of The Royal College of Paediatrics and Child Health, could perhaps be reached. Topics should include the withdrawal of ineffective care (not prohibited by the Roman Catholic church), the institution of palliative care, and greater involvement of parents and independent witnesses in decision making. The legal status of neonatal euthanasia is uniform within Europe (including the Netherlands) at present. This could provide a starting point for wider consultation, and discussion, and eventually the development of a legal framework within Europe.

Arch Dis Child Fetal Neonatal Ed 2004;89:F3

Correspondence to: R W Cooke; Neonatal Unit, Liverpool Women's Hospital Crown Street, Liverpool, L8 7SS; mc19@liv.ac.uk

an intravenous drug offers a cleaner pharmacokinetic study that can further our knowledge of paracetamol pharmacokinetics in premature and term neonates. ${ }^{1-5}$ Allegaert et al are to be congratulated for investigating propacetamol use in premature neonates. Neonatal pharmacokinetic studies are fraught with pitfalls.

Clearance often increases rapidly after birth (postnatal age) no matter what the gestational age at birth. This study gets around this by studying all infants in the first day of life and stratifies by gestational age. It is difficult to enrol neonates of different gestational ages into a longitudinal study. Population modelling-for example, NONMEM software-offers great potential here. 